\title{
RANDOM STURM-LIOUVILLE OPERATORS WITH GENERALIZED POINT INTERACTIONS
}

\author{
DAVid DAMAnik, RAFAel Del Rio AND ASAF L. FRAnCo
}

\begin{abstract}
In this work we study the point spectra of selfadjoint Sturm-Liouville operators with generalized point interactions, where the two one-sided limits of the solution data are related via a general $\operatorname{SL}(2, \mathbb{R})$ matrix. We are particularly interested in the stability of eigenvalues with respect to the variation of the parameters of the interaction matrix. As a particular application to the case of random generalized point interactions we establish a version of Pastur's theorem, stating that except for degenerate cases, any given energy is an eigenvalue only with probability zero. For this result, independence is important but identical distribution is not required, and hence our result extends Pastur's theorem from the ergodic setting to the non-ergodic setting.
\end{abstract}

Mathematics subject classification (2010): 34L05, 47E05, 47N99.

Keywords and phrases: Sturm-Liouville operators, point interactions, eigenvalue problem.

\section{REFERENCES}

[1] S. AlbeVerio, L. DA̧BRows Ki AND P. Kurasov, Symmetries of Schrödinger operators with point interactions, Lett. Math. Phys., 45 (1): 33-47, 1998.

[2] F. V. AtKinson,, Discrete and continuous boundary problems, Mathematics in Science and Engineering, Vol. 8, Academic Press, New York-London, 1964.

[3] D. Buschmann, G. Stolz and J. Weidmann, One-dimensional Schrödinger operators with local point interactions, J. Reine Angew. Math., 467: 169-186, 1995.

[4] R. Del Rio And A. L. Franco, Random Sturm-Liouville Operators with Point Interactions, arXiv e-prints, page arXiv:1903.02714, Mar 2019.

[5] W. N. Everitt, C. Shubin, G. Stolz And A. Zettl, Sturm-Liouville problems with an infinite number of interior singularities, In Spectral theory and computational methods of Sturm-Liouville problems (Knoxville, TN, 1996), vol. 191 of Lecture Notes in Pure and Appl. Math., pages 211-249, Dekker, New York, 1997.

[6] F. GesZTESY AND W. KIRSCH,, One-dimensional Schrödinger operators with interactions singular on a discrete set, J. Reine Angew. Math., 362: 28-50, 1985.

[7] Philip Hartman, Ordinary differential equations, Birkhäuser, Boston, Mass., second edition, 1982.

[8] Don Hinton, Sturm's 1836 oscillation results evolution of the theory, In Sturm-Liouville theory, pages 1-27, Birkhäuser, Basel, 2005.

[9] Serge Lang, $\mathrm{SL}_{2}(\mathbf{R})$, volume 105 of Graduate Texts in Mathematics, Springer-Verlag, New York, 1985 , reprint of the 1975 edition.

[10] Konstantin PankrashKin, Resolvents of self-adjoint extensions with mixed boundary conditions, Rep. Math. Phys., 58 (2): 207-221, 2006.

[11] L. A. PASTuR, Spectral properties of disordered systems in the one-body approximation, Comm. Math. Phys., 75 (2): 179-196, 1980.

[12] LeOnid PAStur AND AleXANDer Figotin, Spectra of random and almost-periodic operators, vol. 297 of Grundlehren der Mathematischen Wissenschaften [Fundamental Principles of Mathematical Sciences], Springer-Verlag, Berlin, 1992.

[13] Walter Rudin, Real and complex analysis, McGraw-Hill Book Co., New York-Toronto, Ont.London, 1966. 
[14] P. ŠEBA, The generalized point interaction in one dimension, Czechoslovak J. Phys. B, 36 (6): 667673, 1986.

[15] Aiping Wang And Anton Zettl, Eigenvalues of Sturm-Liouville problems with discontinuous boundary conditions, Electron. J. Differential Equations, pages Paper No. 127, 27, 2017.

[16] Anton Zettr, Sturm-Liouville theory, vol. 121 of Mathematical Surveys and Monographs, American Mathematical Society, Providence, RI, 2005.

[17] L. A. PASTUR, Spectral properties of disordered systems in the one-body approximation, Comm. Math. Phys., 75 (2): 179-196, 1980.

[18] Joachim Weidmann, Linear operators in Hilbert spaces, Graduate Texts in Mathematics, vol. 68, Springer-Verlag, New York-Berlin, 1980. 